\title{
Bandpass Sampling - An Opportunity To Stress The Importance Of In-Depth Understanding
}

Harold P.E. Stern, Texas State University-San Marcos, USA

\begin{abstract}
Many bandpass signals can be sampled at rates lower than the Nyquist rate, allowing significant practical advantages. Illustrating this phenomenon after discussing (and proving) Shannon's sampling theorem provides a valuable opportunity for an instructor to reinforce the principle that innovation is possible when students strive to have a complete understanding of physical processes and mathematical models.
\end{abstract}

\section{INTRODUCTION}

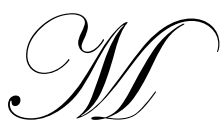

ost engineering students are introduced to Shannon's sampling theorem [1] when they take a course in Signals and Systems, Signal Processing, or Instrumentation. The theorem states:

If a band-limited analog signal $s(t)$ with a maximum frequency $f_{\max } \mathrm{Hz}$ is uniformly sampled at a rate of $f_{s}$ samples/sec, then $s(t)$ can be reconstructed without distortion from the samples provided that the sampling rate $f_{s}>$ $2 f_{\max }$.

The proof of this theorem is simple and elegant, offering the instructor an opportunity to impress upon students the value of using frequency domain mathematics and of being able to envision a signal both in the time domain and the frequency domain. Unfortunately, after completing the proof, many instructors miss a valuable opportunity to show students how the analysis can be taken one step further to reveal cases where certain bandpass signals can be reconstructed without distortion using a sampling rate significantly below $2 f_{\max }$. The lower sampling rate has many practical advantages (less expensive samplers, less required storage, lower transmission speeds) and it also serves to reinforce to students that innovation is possible when students strive to have a complete understanding of physical processes and mathematical models. This paper presents the "extra step" of bandpass sampling and discusses its educational significance.

\section{REVIEW OF SHANNON'S SAMPLING THEOREM}

Let's begin by considering the bandlimited periodic signal $s(t)$ shown in Figure 1(a). This signal is the sum of three cosine terms and has the magnitude spectrum shown in Figure 1(b).

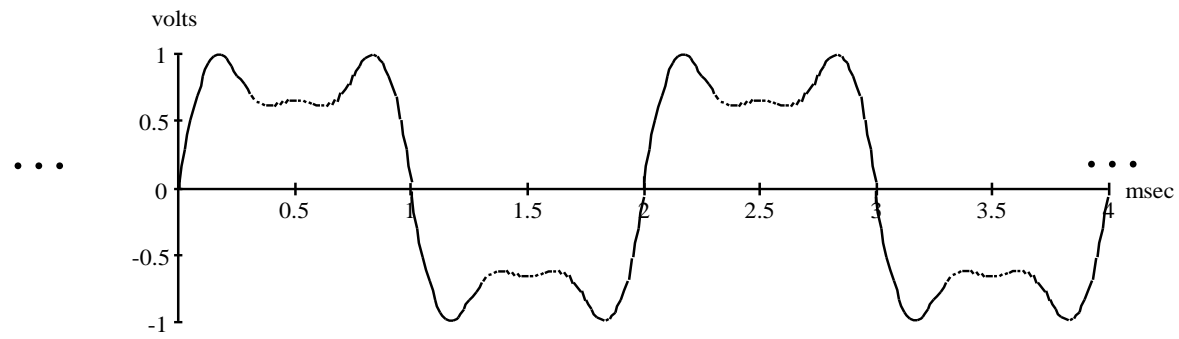

Figure 1a Bandlimited signal $s(t)$ 


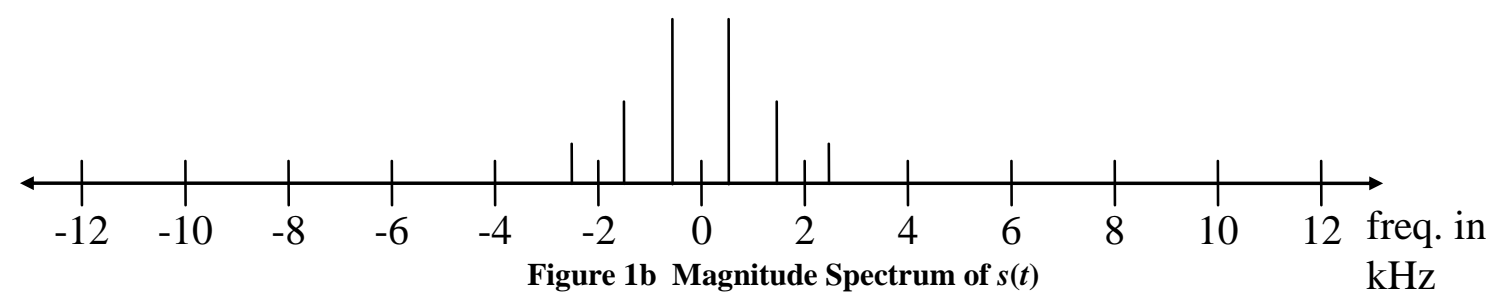

Representing the sampling process as the product of the analog signal $s(t)$ and a series of impulses, we can represent the sampled signal as

$s_{s}(t)=s(t) \sum_{k=0}^{\infty} \delta\left(t-k T_{s}\right)$

where $T_{\mathrm{s}}$ represents the time between samples, which is the inverse of the sampling rate $f_{s}$. Expressing the signal in the frequency domain yields

$$
S_{s}(f)=S(f) * \mathfrak{T}\left\{\sum_{k=0}^{\infty} \delta\left(t-k T_{s}\right)\right\}=S(f) * \sum_{k=-\infty}^{\infty} \delta\left(f-k f_{s}\right)
$$

and the magnitude spectrum of the sampled signal is shown in Figure 2 (sampling rate $f_{s}$ is arbitrarily set at 8000 samples/sec).

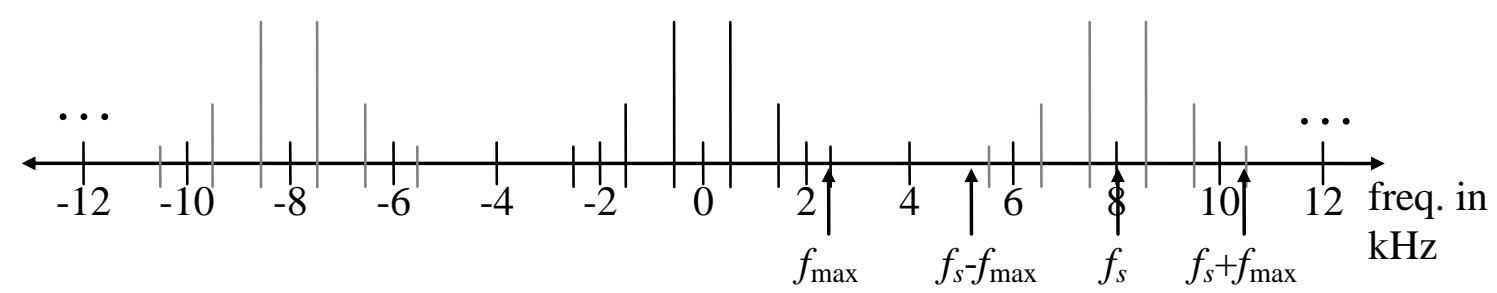

Figure 2 Magnitude Spectrum of $s_{s}(t)$ with $f_{s}=8000$ samples/sec

Comparing Figure 2 to Figure 1 shows that the spectrum of the sampled signal contains the spectrum of the original signal plus extra copies of the spectrum of the original signal (known as aliases), centered at integral multiples of the sampling frequency. The original spectrum can be recovered without the aliases by using a lowpass filter with a cutoff frequency of $f_{\max }$, provided that the spectra of none of the aliases overlap the spectrum of the original signal. This condition is equivalent to saying that the lowest frequency in the spectrum of the alias closest to original signal $\left(f_{s}-f_{\max }\right)$ is greater than the maximum frequency in the spectrum of the original signal $\left(f_{\max }\right)$, or, equivalently, $f_{s}>2 f_{\max }$. The value $2 f_{\max }$ is often known as the Nyquist rate. At this point the sampling theorem is proven and practical considerations can be discussed, such as anti-alias filtering and sampling at higher rates to create guardbands between aliases to compensate for nonideal frequency response of the reconstruction filter. In many courses, the subject of sampling is concluded at this point and the students dutifully memorize the Nyquist rate and its application to sampling.

\section{BANDPASS SAMPLING}

The instructor can, however, take the lesson one step further, showing students how a complete understanding of the sampling process can lead to innovation and a significantly improved system design. The instructor can create an example showing students a bandpass signal $x(t)$ with all of its frequency components lying in the band of, say, 80 $\mathrm{kHz}-100 \mathrm{kHz}$. Bandpass signals are common in many types of sensors and in systems using modulated signals. The instructor can then ask students the minimum sampling rate required to allow distortionless reconstruction. Applying 
the Nyquist rate produces a minimum of 200,000 samples/sec, and if 16 bits of quantization are required for each sample, then 3.2 Mbits/sec are required to store the sampled signal or to transmit it in a digital stream. Practical considerations (sampling at higher rates to compensate for nonideal filters) make the required transmission or storage rate even higher.

Stressing that students should go back to a fundamental understanding of the sampling process, the instructor can provide a magnitude spectrum for the bandpass signal, such as Figure 3, and then ask students to draw the spectrum of the sampled signal using the Nyquist rate of 200,000 samples/sec. Figure 4 shows such a spectrum with the various aliases represented by different shadings.

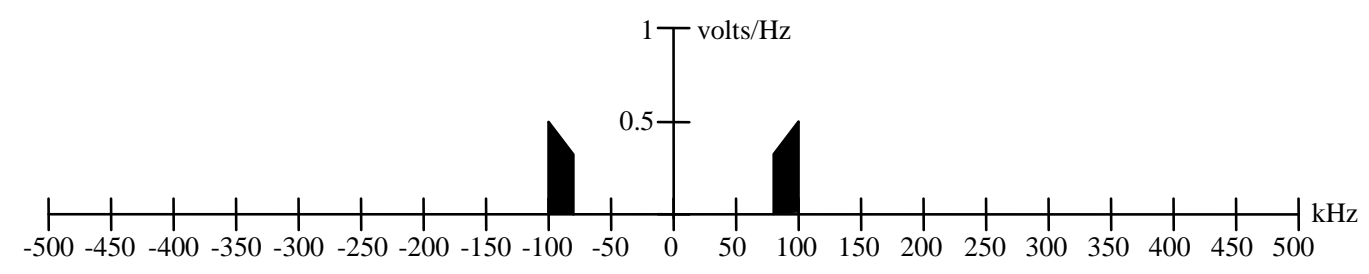

Figure 3 Magnitude Spectrum of Bandpass Signal $x(t)$

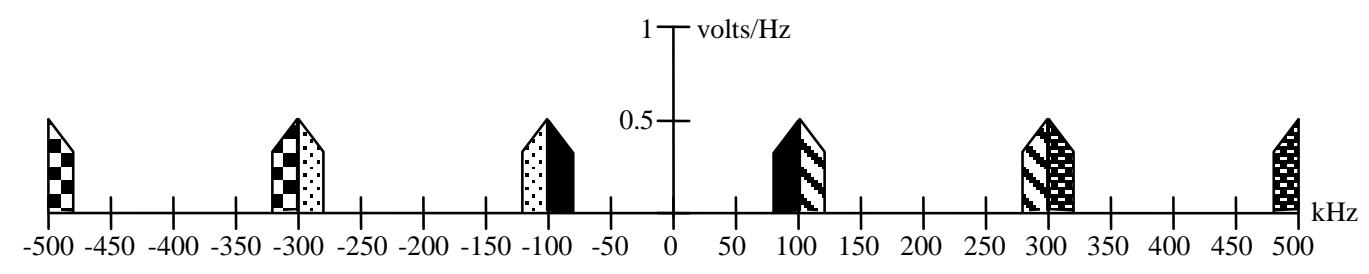

Figure 4 Magnitude Spectrum of Sampled Bandpass Signal $x_{s}(t), f_{s}=200,000$ samples/sec

Examining Figure 4, the original signal can be reconstructed using a baseband filter with a $100 \mathrm{kHz}$ cutoff frequency (as before, a higher sampling rate will compensate for a nonideal frequency response of the filter). At this point, the instructor should note that there is significant open space in the spectrum of the sampled signal. Reminding the students of the fundamentals (that sampling creates aliases whose spectra are centered at integral multiples of the sampling rate) the instructor can now ask the students what will happen if the sampling rate is reduced below the Nyquist rate of 200,000 samples/sec. The answer, of course, is that a slight reduction will cause the aliases closest to the spectrum of the original signal to overlap the spectrum of the original signal, but as the sampling rate is further reduced the overlap will disappear as one side of the alias spectrum slides completely to frequencies below the spectrum of the original signal. The original signal can still be reconstructed from the sampled signal as long as there is no overlap - all that is necessary is a bandpass reconstruction filter with appropriate cutoff frequencies instead of a baseband reconstruction filter. Figure 5 shows the case of sampling at 70,000 samples/sec (only slightly more than $1 / 3$ of the 200,000 sample/sec Nyquist rate). The aliases do not overlap the spectrum of the original signal and the reduction in sampling rate means that the system requires only $35 \%$ of the storage (or streaming transmission speed) required by sampling at the Nyquist rate.

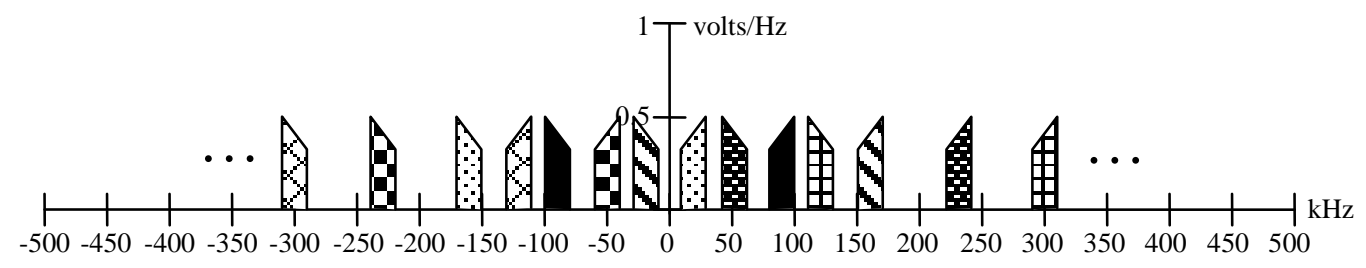

Figure 5 Magnitude Spectrum of Sampled Bandpass Signal $x_{s}(t), f_{s}=70,000$ samples $/ \mathrm{sec}$ 
Reviewing the bandpass sampling process, one can see that the degree of reduction of sampling rate depends on the bandwidth of the signal and the values of the signal's minimum and maximum frequencies (called $f_{l}$ and $f_{2}$, respectively). In order to achieve any reduction, the bandwidth of the signal must be less than $f_{l}$. In bestcase situations, the sampling rate can be reduced toward a lower limit of twice the signal's bandwidth. Reference [2] provides mathematical formalae and plots to show how much of a reduction in sampling rate can be achieved for various values of signal bandwidth, $f_{1}$, and $f_{2}$; however, the conceptual understanding of the process is reinforced if, instead, students draw the spectrum of the sampled signal for various sampling rates and solve the problem geometrically [3].

The instructor can now stress a point much broader than bandpass sampling by discussing how the reduced sampling rate would not have been "discovered" if students merely memorized the Nyquist rate and its proof. Rather, the innovation was "revealed" through a thorough understanding of the sampling process and its associated mathematical models. In our highly competitive global economy, successful engineers will need to be innovators in all aspects of their job, and innovation requires deep understanding of physical processes and the mathematics involved in modeling the processes.

\section{CONCLUSIONS}

As engineering educators, we know that in order for students to succeed as technological innovators they need a thorough understanding of the physical processes and mathematical models of various engineering phenomena. Unfortunately, the rapid pace and large quantity of material being taught in typical courses causes students to often lose sight of the importance of developing in-depth understanding. As instructors, we should embrace opportunities to practically reinforce the value of a thorough understanding. When teaching a Signals and Systems, DSP, or Instrumentation course, bandpass sampling provides such an opportunity.

\section{AUTHOR INFORMATION}

Harold P.E. Stern (BSEE University of Texas at Austin; MSEE U.T. Arlington; Ph.D. U.T. Arlington) is founding director and professor of the Ingram School of Engineering at Texas State University-San Marcos. Prior to joining Texas State, Dr. Stern was a faculty member for 16 years at the University of Alabama in Tuscaloosa. Recipient of three university-wide, three college-wide, and six departmental teaching awards at Alabama, Dr. Stern's research foci are wireless communication systems and engineering education.

\section{REFERENCES}

1. C.E. Shannon, "Communication in the Presence of Noise," Proceedings of the IRE, vol. 37, no 1, pp. 10 21, Jan 1949. Reprinted in Proceedings of the IEEE, Vol 86, no 2, pp. 447-457, Feb. 1998.

2. $\quad$ R.G. Lyons, Understanding Digital Signal Processing, Reading, Mass: Addison-Wesley, 1997.

3. H.P.E. Stern and S.A. Mahmoud, Communication Systems: Analysis and Design, Upper Saddle River, NJ: Prentice-Hall, 2004. 